The Astrophysical Journal, 474:378-388, 1997 January 1

(C) 1997. The American Astronomical Society. All rights reserved. Printed in U.S.A.

\title{
ZERO-ENERGY ROTATING ACCRETION FLOWS NEAR A BLACK HOLE
}

\author{
DONGSU RYU \\ Department of Astronomy and Space Science, Chungnam National University, Daejeon 305-764, Korea; \\ ryu@sirius.chungnam.ac.kr \\ SANDIP K. ChaKRABARTI \\ Tata Institute of Fundamental Research, Mumbai 400 005, India; chakraba@tifrc2.tifr.res.in \\ AND \\ DiEgo MolTENI \\ Istituto di Fisica, Università di Palermo, via Archirafi 36, I-90123 Palermo, Italy; molteni@gifco.fisica.unipa.it \\ Received 1996 May 20; accepted 1996 July 22
}

\begin{abstract}
We characterize the nature of thin, axisymmetric, inviscid accretion flows of cold adiabatic gas with zero specific energy in the vicinity of a black hole by the specific angular momentum. Using twodimensional hydrodynamic simulations in cylindrical geometry, we present various regimes in which the accretion flows behave distinctly differently. When the flow has a small angular momentum $\left(\lambda \lesssim \lambda_{b}\right)$, most of the material is accreted into the black hole, forming a quasi-spherical flow or a simple disklike structure around it. When the flow has a large angular momentum (typically, larger than the marginally bound value, $\lambda \gtrsim \lambda_{\mathrm{mb}}$ ), almost no accretion into the black hole occurs. Instead, the flow produces a stable standing shock with one or more vortices behind it and is deflected away at the shock as a conical, outgoing wind of higher entropy. If the flow has an angular momentum somewhat smaller than $\lambda_{\mathrm{mb}}\left(\lambda_{u} \lesssim \lambda \lesssim \lambda_{\mathrm{mb}}\right)$, a fraction (typically $5 \%-10 \%$ ) of the incoming material is accreted into the black hole, but the flow structure formed is similar to that for $\lambda \gtrsim \lambda_{\mathrm{mb}}$. Some of the deflected material is accreted back into the black hole while the rest is blown away as an outgoing wind. These two cases with $\lambda \gtrsim \lambda_{u}$ correspond those studied in the previous works by Molteni, Lanzafame, \& Chakrabarti and Ryu et al. However, the flow with angular momentum close to the marginally stable value $\left(\lambda_{\mathrm{ms}}\right)$ is found to be unstable. More specifically, if $\lambda_{b} \lesssim \lambda \sim \lambda_{\mathrm{ms}} \lesssim \lambda_{u}$, the flow displays a distinct periodicity in the sense that the inner part of the disk is built and destroyed regularly. The period is roughly equal to (4-6) $\times 10^{3} R_{g} / c$, depending on the angular momentum of the flow. In this case, the internal energy of the flow around the black hole becomes maximum when the structure with the accretion shock and vortices is fully developed. But the mass accretion rate into the black hole reaches a maximum value when the structure collapses. Averaged over periods, more than half the incoming material is accreted into the black hole. We suggest the physical origin of these separate regimes from a global perspective. Then we discuss the possible relevance of the instability work to quasi-periodic oscillations.
\end{abstract}

Subject headings: accretion, accretion disks - black hole physics - hydrodynamics — shock waves

\section{INTRODUCTION}

Accretion onto compact objects is an important ingredient in many astrophysical systems that involve mass transfer from one object to another, such as in binary systems, or from the surrounding medium to the center object, such as in active galactic nuclei, and hence has been the topic of many studies, both analytic and numerical. Bondi (1952) first considered the accretion of adiabatic gas into a gravitating point mass, $M_{\mathrm{bh}}$, with the assumptions of nonrelativistic (but Newtonian) treatment and spherical symmetry, and found a shock-free solution with a steady state accretion rate given by

$$
\dot{M}_{\mathrm{acc}}=4 \pi \alpha\left(G M_{\mathrm{bh}}\right)^{2} \rho / c_{s}^{3},
$$

where $\rho$ and $c_{s}$ are the density and sound speed of the gas at infinity. Here $\alpha$ is a numerical constant of order unity.

Studies of more realistic accretion of gas with a finite amount of angular momentum, however, indicated the existence of the variety and complexity of structures that form around the central object. Hawley and collaborators (Hawley, Smarr, \& Wilson 1984a, 1984b; Hawley 1986; Hawley \& Smarr 1986) demonstrated through numerical simulations that, as accretion flow approaches the central object, it encounters an increasing centrifugal force, and material is reflected away before reaching the black hole's horizon if its angular momentum is sufficiently great. In their simulations, the accretion shock where the material is deflected is nonsteady and travels outward.

The full behavior of accretion flows with a finite amount of angular momentum (both for optically thick and optically thin flows) was understood later through analytic work on basically one-dimensional flows which are in vertical equilibrium (Chakrabarti 1989, 1990). There it was shown that two parameters, namely, specific energy and specific angular momentum, are required to define the critical accretion rates. It was also observed that there is a large region of the parameter space spanned by the specific energy and the specific angular momentum of the flows in which the accretion shock could be perfectly stable. These results were verified later with one-dimensional and twodimensional numerical simulations based on the smoothed particle hydrodynamics (SPH) and total variation diminishing (TVD) methods (Chakrabarti \& Molteni 1993; Molteni, Lanzafame, \& Chakrabarti 1994; Ryu et al. 1995, hereafter Paper I; Molteni, Ryu, \& Chakrabarti 1996a). These works showed that weakly viscous, quasi-spherical accretion (e.g., the optically thick accretion disks of Paczyński \& Wiita 
1980 or optically thin ion tori of Rees et al. 1982) can first form a giant thick disk and, close to the black hole, another, smaller, thick disk in the postshock region (if the shock exists). The accretion is accompanied by a conical, outgoing wind depending on the geometry and the specific angular momentum of incoming flow. In conformity with the analytic work, shock-free flows passing only through the inner or outer sonic point were also found to be stable at least in one dimension.

Furthermore, it was shown that the accretion shock may continue to persist in weakly or strongly viscous flows, depending on viscosity prescriptions (Chakrabarti 1990; Chakrabarti \& Molteni 1995), if the angular momentum at the outer boundary is kept sub-Keplerian (though the result is not certainly restricted to this outer boundary condition). On the other hand, two-dimensional simulations of strongly viscous Keplerian disks found no accretion shock (Eggum, Coroniti, \& Katz 1985, 1987, 1988). Recently, Igumenshchev, Chen, \& Abramowicz (1996) and Manmoto et al. (1996) have studied the numerical simulations of advection-dominated flows (Narayan \& Yi 1994). Since the basic equations solved were similar to those in Chakrabarti \& Molteni (1995), these works also showed the redistribution of angular momentum (Igumenshchev et al. 1996) and disappearance of the shock at high viscosity (Manmoto et al. 1996) that was shown in Chakrabarti \& Molteni (1995). This is consistent with the understanding that advection-dominated flows constitute a special case of viscous advective disks (see Chakrabarti 1996). The general behavior in the latter flows is that the parameter space that forms standing shock waves "shrinks" as viscosity is increased, and beyond a critical viscosity, the shock disappears and a Keplerian disk forms that passes through the inner sonic point.

The structure with an accretion shock around the black hole could be unstable in some situations. We demonstrated in Paper I that the accretion flow with a large angular momentum $\left(\lambda \gg \lambda_{\mathrm{mb}}\right)$ is unstable if the incoming flow has a small thickness. Molteni, Sponholz, \& Chakrabarti (1996b) showed that accretion flow can be unstable if cooling is nonnegligible and its timescale is comparable to the dynamical timescale of the postshock flow. These instabilities, either dynamically induced as in Paper I or thermally induced as in Molteni et al. (1996b), result in the oscillatory behavior of the structure formed around the black hole, causing the energy output from it to vary quasiperiodically. These oscillating disks may be capable of producing the right frequencies and amplitude modulations for the quasi-periodic oscillation (QPO) phenomenon seen in wide variety of objects such as low-mass X-ray binaries (e.g., van der Klis 1989; Dotani 1992) and white dwarf candidates (e.g., Mauche 1995, 1996), as well as in active galaxies (e.g., Halpern \& Marshall 1996).

In Paper I, we considered the properties of thin, rotating accretion flows near a Newtonian point mass. The angular momentum used was larger than the marginally bound value $\left(\lambda_{\mathrm{mb}}=2 R_{g} c\right.$, where $R_{g}$ is the Schwarzschild radius). We obtained a structure with shocks and winds consistent with the expectations from earlier work in this angular momentum regime (Hawley \& Smarr 1986; Molteni et al. 1994). As an extension of Paper I, in this paper we study the properties of thin, rotating accretion flows around a black hole. Here we deal with transonic flows, which can have an extra saddle-type sonic point just outside the horizon
(Liang \& Thompson 1980; Chakrabarti 1989, 1990) through which the material can accrete as well. We focus especially on the cold flows (before shocking, if a shock exists) that are at rest at infinity, i.e., cold flows with negligible net energy. Then whether a disk will be accreting at all or whether a disk will include a stationary/nonstationary shock will depend only on a single parameter, namely, the specific angular momentum of the flows. Here we demonstrate that accretion flows with angular momentum somewhat larger or smaller than the marginally stable value $\left[\lambda_{\mathrm{ms}}=(3 / 2)^{3 / 2} R_{g} c \equiv 1.837 R_{g} c\right.$ for a Schwarzschild black hole] establish a stable structure around the black hole, while flows with angular momentum close to $\lambda_{\mathrm{ms}}$ show an unstable behavior, exhibiting periodic construction and destruction of the inner accretion disk. We anticipate that this regime of "unstable flows" could be very important from astrophysical points of view, as radiation emitted from this region could be periodically modulated, producing a QPO behavior, particularly given that the frequency and amplitude of the modulation are similar to the corresponding observed values.

The plan of the paper is as follows: In $\S 2$, we describe the problem by defining the normalization units and identifying the free parameters. We then demonstrate the existence of different angular momentum regimes, in which accretion flows are expected to behave differently, using an analytic consideration. In $\S 3$, we describe briefly the numerical scheme and present the results of numerical simulations for accretion flows with different angular momenta. Then we suggest the physical origin of the behavior of the accretion flows seen in the numerical calculations. We show that the region of the parameter space in which such unstable behavior is seen actually corresponds to the region where two saddle-type sonic points exist but the stationary shock condition is not satisfied (Chakrabarti 1989). Finally, in $\S 4$, we discuss astrophysical implications of this work and make concluding remarks.

\section{FORMULATION OF THE PROBLEM}

We consider the hydrodynamics of axisymmetric accretion flows under the influence of a black hole with mass $M_{\mathrm{bh}}$ located at the center. We choose cylindrical coordinates $(r, \theta, z)$. We assume that the gravitational field of the black hole can be described in terms of the potential introduced by Paczyński \& Wiita (1980),

$$
\phi(r, z)=-\frac{G M_{\mathrm{bh}}}{R-R_{g}}
$$

where $R=\left(r^{2}+z^{2}\right)^{1 / 2}$ and the Schwarzschild radius is $R_{g}=2 G M_{\mathrm{bh}} / c^{2}$. The gas mass in the accreting flow is assumed to be much smaller than the black hole's mass, so the self-gravity of the gas is negligible compared to the gravity of the black hole. In addition, the accreting matter is assumed to be adiabatic gas, without cooling and dissipation, and is described by a polytropic equation of state, $P=K \rho^{\gamma}$, where $\gamma$ is the adiabatic index, which is considered to be constant at $4 / 3$ throughout the flow. $K$ is related to the specific entropy of the flow, $s$, and varies only at shocks (if present). Since we consider only the weak-viscosity limit, the specific angular momentum of the accretion flow, $\lambda=$ $r v_{\theta}$, is assumed to be conserved. With these settings, the problem has three intrinsic quantities, the black hole mass $M_{\mathrm{bh}}$, the speed of light $c$, and the Schwarzschild radius $R_{g}$. 
In what follows, we use these as the units of mass, velocity, and length, respectively.

With this normalization, the condition of the incoming gas can be characterized by a minimum of the following four parameters at a point distant from the black hole (or the outer boundary of the domain of numerical calculations, $r_{b}$; see $\S 3$ ): (1) the radial velocity, $v_{r}$, (2) the angular momentum, $\lambda$, (3) the sound speed, $a$, and (4) the geometry (or, more specifically, the thickness, $h_{\text {in }}$, of the inflow), if the complication of the vertical density structure is ignored and the vertical velocity, $v_{z}$, is negligible.

The above number of free parameters is still too large to explore with numerical simulations, and we reduce it with the following further assumptions: First, we consider the gas to be at rest with negligible pressure at infinity and start to accrete into the black hole. So we assume that the flows have zero total energy at the outer boundary. This assumption implies that, for a given specific angular momentum, $v_{r}$ and $a$ are not independent but are related by the condition

$$
\mathscr{E}=\frac{v_{r}^{2}}{2}+\frac{a^{2}}{\gamma-1}+\frac{\lambda^{2}}{2 r^{2}}+\phi=0
$$

with negligible $v_{z}$. Second, we consider the incoming gas at the outer boundary to be cool and hence supersonic. We assume a fixed Mach number $M=v_{r} / a$ that is large $(M \gg 1)$ at the outer boundary. Finally, we consider the inflow at the outer boundary to have a small thickness or a small arc angle $\theta=\arctan \left(h_{\mathrm{in}} / r_{b}\right) \ll 1$. With these assumptions, we can fix three parameters, $v_{r}, a$, and $h$, and are effectively left with a single free parameter, the specific angular momentum $\lambda$, using which we classify the properties of accretion flows.

In order to understand the role of angular momentum, we note that whereas in a Newtonian geometry a particle with any nonzero angular momentum faces an infinite potential barrier close to the symmetry axis, around a black hole the particle "starts feeling" the barrier only if $\lambda>\lambda_{\mathrm{ms}}$, the marginally stable angular momentum. This is the minimum angular momentum that is required by a rotating particle to balance the gravity of the black hole anywhere outside it. The vanishing of the net force (the centrifugal plus gravitational forces),

$$
f=\frac{\lambda^{2}}{r^{3}}-\frac{1}{2(r-1)^{2}}
$$

defines the Keplerian angular momentum, $\lambda_{\text {Kep }}$, at a distance $r=r_{\text {eq }}$. For an accretion flow with $\lambda>\lambda_{\mathrm{ms}}$, there are two values of $r_{\text {eq }}$. Between them, the centrifugal force dominates over the gravitational force, so the total force is directed outward. Outside them, the gravitational force is larger, so the total force is directed inward. If a flow approaches from infinity, it feels the inward force at first and then feels the outward force. Hence the flow can form a stable disk around the black hole. But if the accretion flow has $\lambda<\lambda_{\mathrm{ms}}$, the gravitational force always dominates over the centrifugal force, so the flow always feels the inward attraction. This flow thus cannot form a stable disk but rather accretes directly into the black hole in a quasispherical manner.

The marginally bound angular momentum, $\lambda_{\mathrm{mb}}$, on the other hand, defines the highest possible angular momentum with which a particle at rest at infinity can be accreted into a black hole (see, e.g., Shapiro \& Teukolsky 1983). Or, inversely, it is the smallest possible angular momentum for a rotating particle to bounce back to infinity. The location of bouncing back as a function of $r$ from the black hole is commonly known as the funnel wall and can be found from the vanishing of the energy in equation (3) (see, e.g., Hawley $\&$ Smarr 1986). Along the equatorial plane, it is

$$
\mathscr{E}=\frac{\lambda^{2}}{2 r^{2}}-\frac{1}{2(r-1)}=0 .
$$

We denote the radius of the funnel wall along the equatorial plane by $r_{f}$. If $\lambda>\lambda_{\text {mb }}$, flows are required to be hot $(\mathscr{E}>0)$ in order to accrete onto a black hole.

In Figure 1, we show $r_{\text {eq }}$ and $r_{f}$ as long-dashed and shortdashed curves, respectively, and also note the limiting angular momenta. Since we are interested in the transonic properties of accretion flows, we also show the locations of the sonic points $\left(r_{\text {sonic }} ;\right.$ dotted curve) of zero-energy flows, calculated by using the standard analysis of onedimensional flows (Chakrabarti 1989, 1990; Chakrabarti \& Molteni 1993). With $\lambda<\lambda_{b}(=1.782)$, accretion flows have no sonic point at a finite distance. With $\lambda_{b} \leq \lambda \leq \lambda_{\mathrm{mb}}$, they have a saddle-type inner sonic point, $r_{s}$ (dotted curve with negative slope), as well as an unphysical center-type sonic point, $r_{c}$ (dotted curve with positive slope). With $\lambda>\lambda_{\mathrm{mb}}$, they have only the unphysical sonic point.

Furthermore, we can classify the angular momentum space into four zones, with three boundaries at $\lambda_{b}=1.782$, $\lambda_{u}=1.854$, and $\lambda_{\mathrm{mb}}=2$. For $\lambda<\lambda_{b}$ (zone I), with no sonic point at a finite distance, only supersonic flows can accrete onto a black hole without accompanying a shock. In the region $\lambda_{u}<\lambda<\lambda_{\mathrm{mb}}$ (zone III), the Rankine-Hugoniot condition for shock formation is satisfied at $r>r_{s}$ if the inflow is supersonic (Chakrabarti 1989). Thus supersonic flows can settle into a stable solution that includes a shock. However, in the region $\lambda_{b}<\lambda<\lambda_{u}$ (zone II), the Rankine-Hugoniot condition is not satisfied. Therefore supersonic flows cannot settle into a stable solution. For $\lambda>\lambda_{\mathrm{mb}}$ (zone IV), the inner

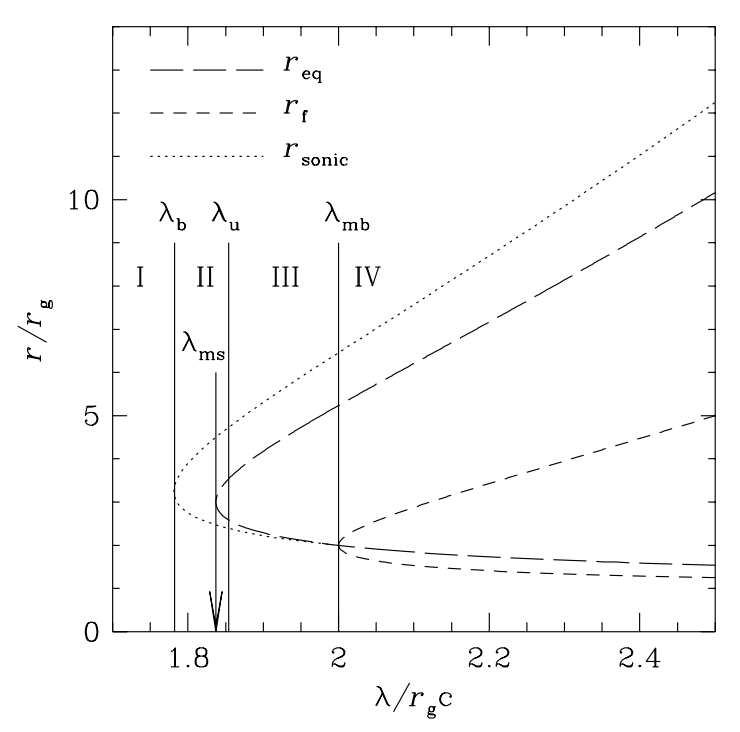

FIG. 1.-Radius of equal gravitational and centrifugal forces, $r_{\text {eq }}$, radius of the funnel wall along the equatorial plane, $r_{f}$, and the locations of the sonic points of one-dimensional flows, $r_{\text {sonic }}$, as functions of specific angular momentum. The angular momentum space is divided into four zones according to whether sonic points and shocks exist or not. Also, the locations of important angular momenta are marked. 
sonic point does not exist. Thus, although a standing shock may form if the inflow is supersonic, matter cannot accrete onto a black hole but must be diverted as a wind. Although the above classification is based on the analysis for onedimensional flows, we find through numerical simulations that these subdivisions may be robust even in twodimensional flows. What is more, we discover that the flows in zone II exhibit a periodic behavior, virtually building and destroying the whole disk structure while constantly trying to form a standing shock wave, but never succeeding.

The classification described above is applicable to the flows of zero net energy and adiabatic index of $4 / 3$. It can be extended to more general flows with nonzero energy and any adiabatic index. Thus, for instance, the unstable periodic behavior will be present in the region of the parameter space in which the shock condition is not satisfied, though the flow has two saddle-type sonic points (Fig. 4 of Chakrabarti 1989). Details of the general classification with relevant numerical simulations will be presented elsewhere (Chakrabarti, Ryu, \& Molteni 1996).

\section{RESULTS OF NUMERICAL CALCULATIONS}

\subsection{Numerical Method and Setup}

The numerical calculations have been carried out with a hydrodynamic code based on the TVD scheme, originally developed by Harten (1983). It is an explicit, second-orderaccurate scheme that is designed to solve a hyperbolic system of conservation equations, like the system of hydrodynamic equations. It is a nonlinear scheme obtained by first modifying the flux function and then applying a nonoscillatory first-order-accurate scheme to obtain a resulting second-order accuracy. The key merit of this scheme is that it achieves the high resolution of a second-order accuracy while preserving the robustness of a nonoscillatory firstorder scheme.

Harten (1983) described the application of his scheme to the set of one-dimensional hydrodynamic equations in Cartesian geometry and presented some tests including shock capturing. The version of the TVD code used in this paper is the two-dimensional one in cylindrical geometry that was described in detail in Paper I, except for the form of the gravitational force. Here we use the force that is derived from the modified potential in equation (2). In Molteni et al. (1996a), the code was tested for the problem of onedimensional and two-dimensional accretion flows by comparing the solutions from the code with the analytic solutions and the numerical solutions from the SPH code and demonstrated to be sufficiently accurate for the numerical study of accretion flows.

The equations solved numerically with the TVD code are given in Molteni et al. (1996a). Note that, in the case of an axisymmetric flow without viscosity, the equation for azimuthal momentum states simply the conservation of specific angular momentum,

$$
d \lambda / d t=0 .
$$

So it can be decoupled from the rest of the hydrodynamic equations if the flow has a uniform angular momentum. But in the problem considered in this paper, the background material (of relatively low density) and the accreting material may have different angular momenta and be mixed in some cells, resulting in a variation in specific angular momentum. So the code used in this paper solves the whole set of the hydrodynamic equations, including that for azimuthal momentum. As a result, however, the angular momentum of the accreting material is not conserved exactly. The error in the angular momentum conservation is typically less than $\sim 2 \%$ in the material approaching the black hole, but could be up to $\sim 5 \%$ in the material bounced back as a wind. For a discussion of the possible effects of this error, see Molteni et al.(1996a).

The calculations were performed in a setting similar to that used in Paper I. The computational box occupies one quadrant of the $r-z$ plane with $0 \leq r \leq 50$ and $0 \leq z \leq 50$. The incoming gas enters the box through the outer boundary, located at $r_{b}=50$. We chose the adiabatic index $\gamma=4 / 3$ since it is appropriate for a wide range of astrophysical circumstances, such as relativistic flows, optically thick radiation-dominated disks, and optically thin flows with cooling effects (such as the Comptonization of external soft photons). We have chosen the density of the incoming gas $\rho_{\text {in }}=1$ for convenience since, in the absence of selfgravity and cooling, the density is scaled out, rendering the simulation results valid for any accretion rate.

We have considered accretion flows with angular momentum $1.5 \leq \lambda \leq 2.25$ covering all four zones in Figure 1. The incoming flow at the outer boundary is directed toward the symmetry axis along the negative $r$-axis with $v_{z}=0$. It has a large Mach number, $M=10$, at the outer boundary. This setting, with the assumption of zero total energy in equation (3), fixes the values of $v_{r}$ and $a$ at the outer boundary for the flows with given angular momentum. The incoming flow has been further assumed to be thin with a uniform vertical density. So at the outer boundary, $r_{b}=50 R_{g}$, we inject matter for $0<\theta<\arctan (1 / 10)$. This gives the half-thickness of the incoming flow as $h_{\text {in }}=5 R_{g}$.

In order to mimic the horizon of the black hole at the Schwarzschild radius, we placed an absorbing inner boundary at $R=1.5 R_{g}$, inside which all material is completely absorbed into the black hole. For the background material, we used a stationary gas with density $\rho_{\mathrm{bg}}=10^{-6}$ and sound speed (or temperature) the same as that of the incoming material. Hence the incoming material has a pressure $10^{6}$ times larger than that of the background material. So, once the incoming flow enters through the outer boundary, it initially expands vertically.

Table 1 summarizes the values of the parameters used for the numerical calculations along with the identification of the model number. The duration of each model calculation is indicated as $t_{\text {end }}$ in units of $R_{g} / c$. Except for model M9, all the calculations were performed with $256 \times 256$ cells, so each grid has a size of 0.195 in units of the Schwarzschild radius. The calculation for model M9 was done with $128 \times 128$ cells to study the convergence of our calculations.

\subsection{Numerical Solutions}

Figure 2 shows the temporal evolution of the position of the accretion shock, $r_{\mathrm{sh}}$, along the equatorial plane with $z=0$, the mass accretion rate into the black hole, $\dot{M}_{\text {acc }}$, the mean density, $\bar{\rho}$, and the mean thermal energy, $\bar{e}_{\mathrm{th}}$, of disk matter inside the computational domain. Here $\dot{M}_{\mathrm{in}}$ is the mass inflow rate at the outer boundary. The shock position along the equatorial plan has been calculated in the region with $r \geq 3$.2. Just for the sake of drawing the figure, if there is no shock or a shock in $r<3.2$, the shock position has been set to be 3.2. Figure $2 a$ is for model M2, belonging 

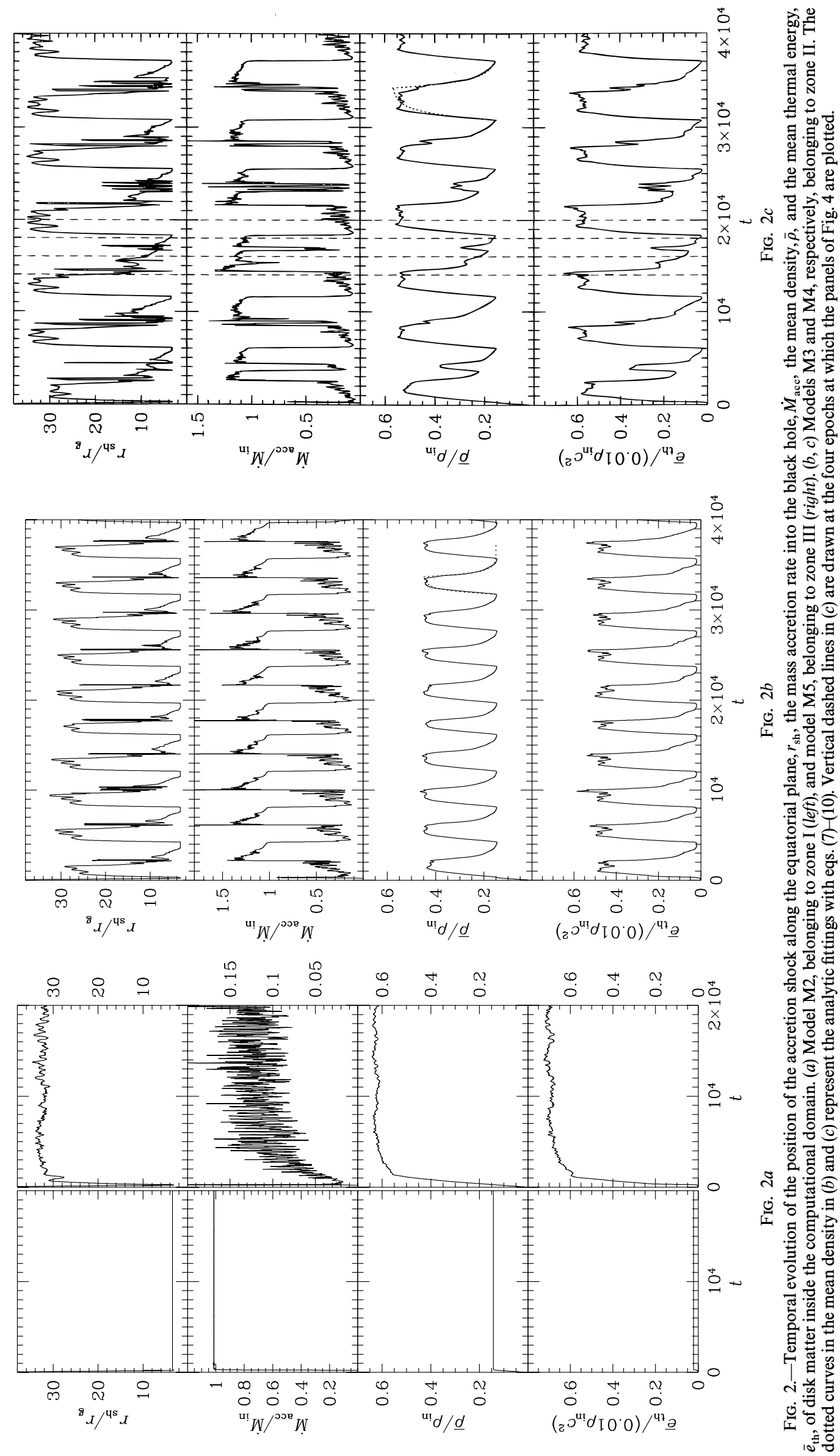
TABLE 1

SUMMARY OF Simulations RePORTED

\begin{tabular}{|c|c|c|c|c|c|}
\hline Model & $\lambda$ & Resolution & $v_{r}$ & $\begin{array}{c}t_{\text {end }} \\
\left(\times 10^{4}\right)\end{array}$ & Comments \\
\hline \multicolumn{6}{|l|}{ Zone I: } \\
\hline M1...... & 1.5 & $256 \times 256$ & 0.13566 & 2 & \multirow{2}{*}{$\begin{array}{l}\text { Stable accretion } \\
\text { without wind }\end{array}$} \\
\hline M2 $\ldots \ldots$ & 1.75 & $256 \times 256$ & 0.13453 & 2 & \\
\hline \multicolumn{6}{|l|}{ Zone II: } \\
\hline M3...... & 1.8 & $256 \times 256$ & 0.13428 & 4 & \multirow[t]{2}{*}{ Periodic behavior } \\
\hline M4 ...... & 1.85 & $256 \times 256$ & 0.13402 & 4 & \\
\hline \multicolumn{6}{|l|}{ Zone III: } \\
\hline M5 ...... & 1.9 & $256 \times 256$ & 0.13376 & 2 & \multirow{2}{*}{$\begin{array}{l}\text { Stable structure with } \\
\text { accretion and wind }\end{array}$} \\
\hline M6...... & 1.95 & $256 \times 256$ & 0.13348 & 2 & \\
\hline \multicolumn{6}{|l|}{ Zone IV: } \\
\hline M7 ....... & 2.0 & $256 \times 256$ & 0.13320 & 2 & \multirow{3}{*}{$\begin{array}{l}\text { Stable structure with } \\
\text { wind but without } \\
\text { significant accretion }\end{array}$} \\
\hline $\mathrm{M} 8 \ldots \ldots$ & 2.25 & $256 \times 256$ & 0.13169 & 2 & \\
\hline M9...... & 2.0 & $128 \times 128$ & 0.13320 & 6 & \\
\hline
\end{tabular}

NotE.-All models use $\gamma=4 / 3$ and $\mathscr{E}=0, M=10$ and $h_{\text {in }}=\arctan (1 / 10)$ at the boundary, $r_{b}=50$.

to zone I (left), and model M5, belonging to zone III (right), with $\lambda=1.75$ and 1.9 , respectively. Figure $2 b$ is for model M3 with $\lambda=1.8$ and Figure $2 c$ for model M4 with $\lambda=1.85$, both belonging to zone II. The accretion flow in model M2 shows a stable behavior with virtually all incoming material absorbed into the black hole. The flow in model M5 also shows a stable behavior, but with small aperiodic oscillations. Several different timescales could be identified, but the most dominant one in $\dot{M}_{\text {acc }}$ is $\sim 300$ in our time unit, $R_{g} / c$. But, because of a small amplitude, these oscillations may not have any observational significance. In this model, a small fraction $(\sim 10 \%-15 \%)$ of the incoming material is absorbed into the black hole. This is similar to the result of a thick accretion disk simulation (Molteni et al. 1994). However, the accretion flows in models M3 and M4 show a distinctively periodic behavior, during which the mass accretion rate into the black hole, the disk mass, and the disk thermal energy vary dramatically. The time period of variation is (4-6) $\times 10^{3}$ in our time unit.

Figure 3 shows the density contours and the velocity vectors at $t=2 \times 10^{4}$ in model M2 (left) and model M5 (right). The plots show only the region with $0 \leq r \leq 36$ and
$0 \leq z \leq 36$, although the calculations have been done in the region with $0 \leq r \leq 50$ and $0 \leq z \leq 50$. The density contours are drawn for $\rho=10^{k}$ with $k=-3,2.9,2.8, \ldots, 2$, with the outermost contour near the funnel wall being of lowest density. For clarity, the velocity vectors are drawn at every fourth grid along the $r$ - and $z$-directions. The flow in model M2 with small angular momentum is directly absorbed into the black hole, forming a simple disklike structure around it. The flow in model M5 with large angular momentum forms a stable accretion shock with one or more vortices behind it, as described in our previous work (Molteni et al. 1994; Paper I). The incoming material is deflected at the shock away from the equatorial plane. Some of the deflected material is accreted back into the black hole at a higher latitude, but some is blown away as a conical, outgoing wind.

Figure 4 shows the density contours and the velocity vectors at $t=1.4,1.6,1.8,2 \times 10^{4}$ in model M4. The description of the plotted region and quantities is identical to that of Figure 3. In this case, the structure with an accretion shock and a generally subsonic high-density disk is established around the black hole. However, the structure is

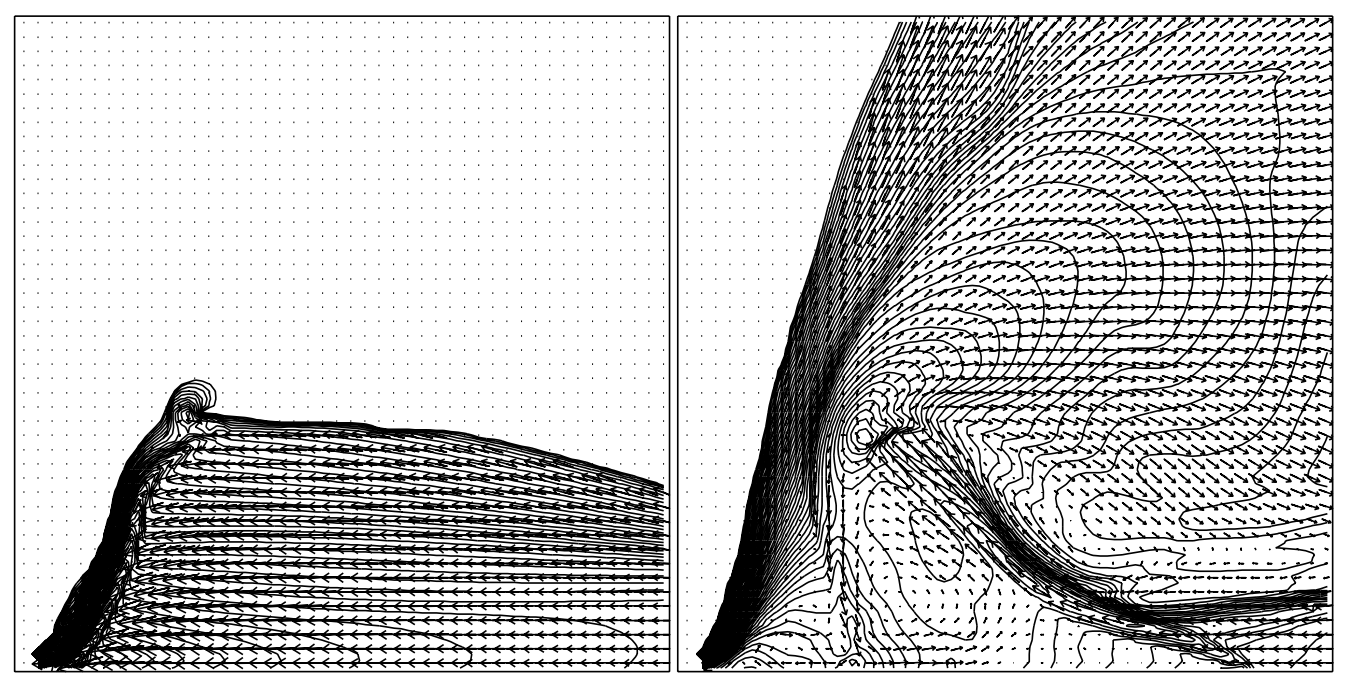

Fig. 3.-Density contours and velocity vectors at $t=2 \times 10^{4}$ in model M2 (left) and model M5 (right). The plots show the region with $0 \leq r \leq 36$ and $0 \leq z \leq 36$. The density contours are drawn for $\rho=10^{k}$ with $k=-3,-2.9,-2.8, \ldots, 2$. The velocity vectors are drawn at every fourth grid along the $r$ - and $z$-directions. 


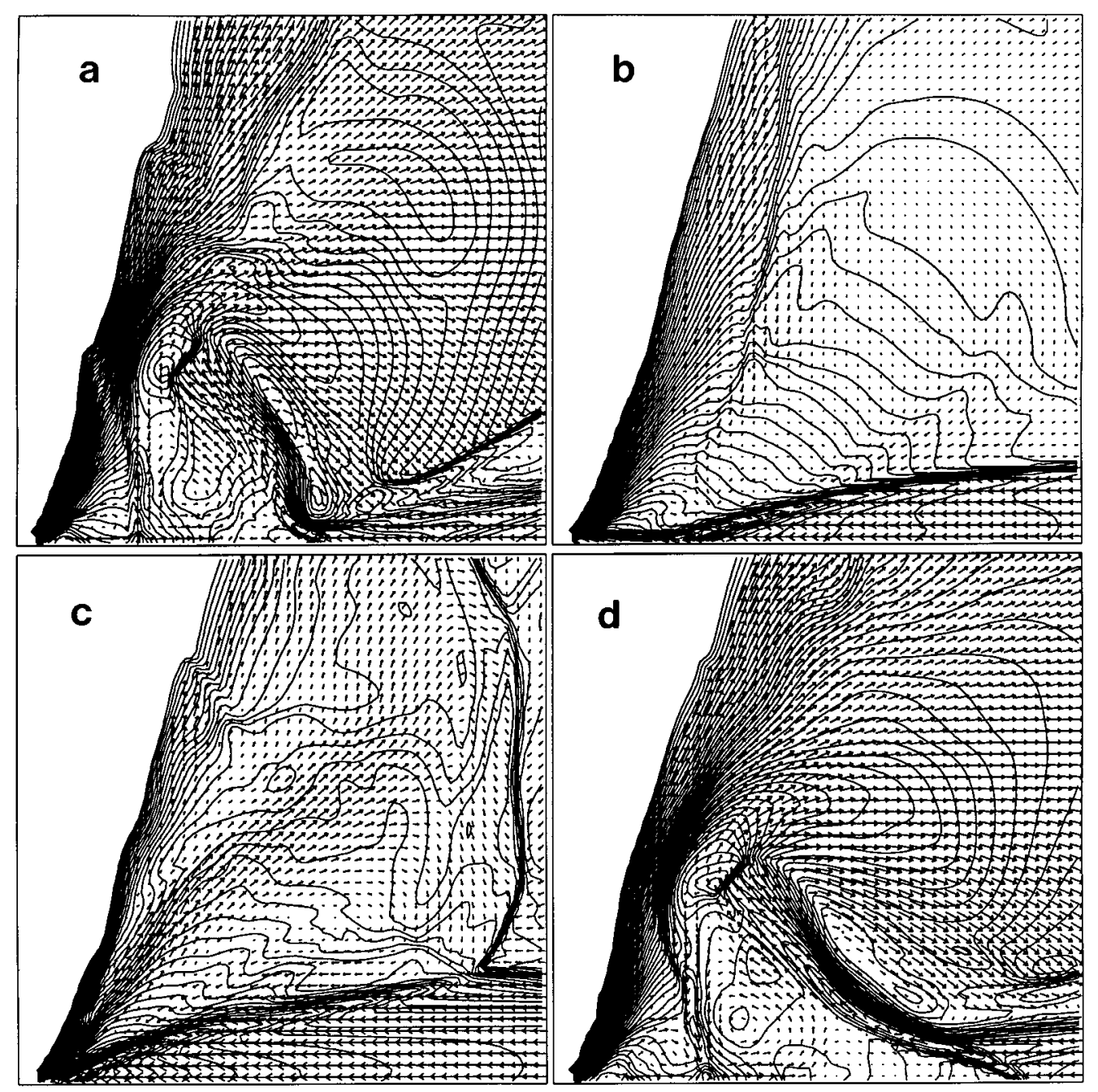

Fig. 4. - Same as Fig. 3, but for $(a) t=1.4 \times 10^{4},(b) t=1.6 \times 10^{4},(c) t=1.8 \times 10^{4}$, and $(d) t=2 \times 10^{4}$ in model M4

not stable. At the accretion shock, the incoming flow is deflected. But some of the postshock flow, which is further accelerated by the pressure gradient behind the shock, goes through a second shock, where the flow is deflected once more downward. The downward flow squeezes the incoming material, and the accretion shock starts to collapse $\left(t=1.4 \times 10^{4}\right.$; see also Fig. $\left.2 c\right)$. In the process of the collapse, some of the postshock material escapes as wind and some is absorbed into the black hole. But all the squeezed incoming material is absorbed into the black hole. Thus the mass accretion rate is larger than the mass inflow rate, $\dot{M}_{\text {acc }} / \dot{M}_{\text {in }}>1$ (the "hyperaccretion stage"). The mass accretion rate becomes highest just after the accretion shock collapses $\left(\sim 1.4 \dot{M}_{\text {in }}\right.$ in model M3 and $\sim 1.2 \dot{M}_{\text {in }}$ in model M4). It then starts decreasing until the shocked material is completely evacuated and $\dot{M}_{\text {acc }} \approx \dot{M}_{\text {in }}$ is reached $\left(t=1.6 \times 10^{4}\right.$ and $t=1.8 \times 10^{4}$; see also Fig. $2 c$ ). After that, the rebuilding of the accretion shock starts with the incoming material bouncing back from the centrifugal barrier. The subsonic postshock region becomes a reservoir of material, so the mass accretion rate is reduced dramatically. With the accumulated material in the postshock region a giant vortex is formed, which in turn supports the accretion shock $\left(t=2 \times 10^{4}\right)$. This is the time when the shock radius and the disk mass are highest but the mass accretion is lowest (see Fig. 2c). This continues until the incoming flow is squeezed enough so that the accretion shock collapses, and the cycle continues. Averaged over periods, more than half the incoming material is accreted into the black hole.

The collapse and rebuilding of the structure take place as a consequence of a very complex and nonlinear interaction of the inflow with the outgoing material, whose property is determined by the accretion shock and the postshock dynamics. As a result, the growth and decay of the average density and pressure follow exponential rules, as in the charging and discharging of a capacitor. In model M3, with $\lambda=1.8$, the average density typically rises as

$$
\bar{\rho}_{r}=0.30[1-\exp (-t / 400)]+0.15,
$$

and falls as

$$
\bar{\rho}_{f}=0.30 \exp (-t / 400)+0.15,
$$

in a single cycle. The full cycle takes about $\tau_{1.8} \approx$ $4 \times 10^{3} R_{g} / c$. In model M4, with $\lambda=1.85$, on the other hand, the average density rises as

$$
\bar{\rho}_{r}=0.42[1-\exp (-t / 750)]+0.15
$$

and falls as

$$
\bar{\rho}_{f}=0.42 \exp (-t / 750)+0.15 .
$$

The full cycle takes about $\tau_{1.85} \approx 6 \times 10^{3} R_{g} / c$. These exponential curves are superposed on density plots in Figures $2 b$ 
and $2 c$. One would have naively expected these timescales to be twice (collapse and expansion) as long as the infall time

$$
\tau_{\text {infall }}=\int_{1}^{r_{\text {out }}} \frac{d r}{v_{r}}
$$

which are 2280 and 3160 , respectively, with

$$
v_{r}=\frac{1}{7}\left(r^{-1}-\lambda^{2} r^{-2}\right)^{1 / 2}
$$

for a zero-energy postshock flow of $\gamma=4 / 3$. Here $r_{\text {out }}$ is the average location of the shock at full expansion. But in reality, it takes almost twice as much, possibly because the backflowing matter has comparable ram pressure, which slows the collapse and expansion significantly.

Figure 5 shows the density contours and the velocity vectors at $t=2 \times 10^{4}$ in model M7 (left) and model M9 (right). The plots show the region with $0 \leq r \leq 50$ and $0 \leq z \leq 50$. The description of the plotted quantities is identical to that of Figure 3. The plots represent the same calculation for the flow in the boundary of zones III and IV but with different resolutions. Overall, they look similar to that of model M5 in zone III (see Fig. 3), although the flow has a much smaller accretion onto the black hole. The general picture agrees with the previous work by Molteni et al. (1994) and in Paper I. Here there is a structure with a stable accretion shock with one or more vortices behind it and a generic outgoing wind for a wide range of angular momenta. Globally, the solutions with two different resolutions look similar to each other, though, while details vary. This indicates that our calculations have converged, at least globally.

Figure 6 shows some quantities of importance as a function of the specific angular momentum. The plots show the time-averaged values of the position of the accretion shock along the equatorial plane, the mass accretion rate into the black hole, the mean density of disk matter, and the mean thermal energy of disk matter. As the specific angular momentum increases, the shock moves farther out and the mass accretion rate decreases, as expected. The disk mass continues to increase with the specific angular momentum. This is because the shock moves out and there is an increasing space where the material is accumulated. However, the disk thermal energy per unit volume reaches a maximum at $\lambda \sim 2$ (and the disk thermal energy per unit mass reaches a maximum at $\lambda \sim 1.95$ ), indicating that the piling up of energy in the disk is most efficient if the flow has $\lambda \sim \lambda_{\mathrm{mb}}$.

\subsection{Comparison with Analytic Solutions}

It is pertinent to ask how the numerical solutions of the time-dependent problem described in $\S 3.2$ compare to the steady state analytic solutions of the similar set of equations. If the flows are strictly one-dimensional without vertical motion, the numerical solutions agree perfectly with the analytic ones, as shown in Molteni et al. (1996a). The flows considered in this paper are, however, two-dimensional, which can expand away from the equator and can form winds as well. The situation becomes more complicated since vortices with backflows can develop and interact with the incoming matter.

In the upper panel of Figure 7, we show the analytic solutions (Mach number vs. radial distance) of onedimensional zero-energy flows with Mach number $M=10$ at $r=50$ whose angular momenta range from $\lambda=1.5$ to 2 . With $\lambda=1.5$ and 1.75 (the uppermost solid and the next short-dash-dotted curves), the flows always remain supersonic $(M>1)$. In these cases, the numerical simulations have found a stable solution (models M1 and M2). Shocks are not expected, if the flow is strictly one-dimensional. Shocks are possible only in a thick flow in vertical equilibrium (see Chakrabarti 1989). We also see a shock formation in the simulations, possibly due to the change in flow geometry through vertical expansion. The short-dashed and the long-dash-dotted curves are for the flows with $\lambda=1.8$ and 1.85 , respectively. Though solution branches through the inner sonic point exist, the upper supersonic branch cannot be connected with the corresponding lower subsonic branch through the shock condition, since the RankineHugoniot conditions are not satisfied. Therefore a steady solution with an accretion shock is impossible. In the simulations (models M3 and M4), we have seen an unstable disk structure with an accretion shock that is constructed and destructed periodically.

In the lower panel of Figure 7, we redraw the analytic Mach number distributions for $\lambda=1.85$ (solid curves) along with the numerical distributions on the equatorial plane

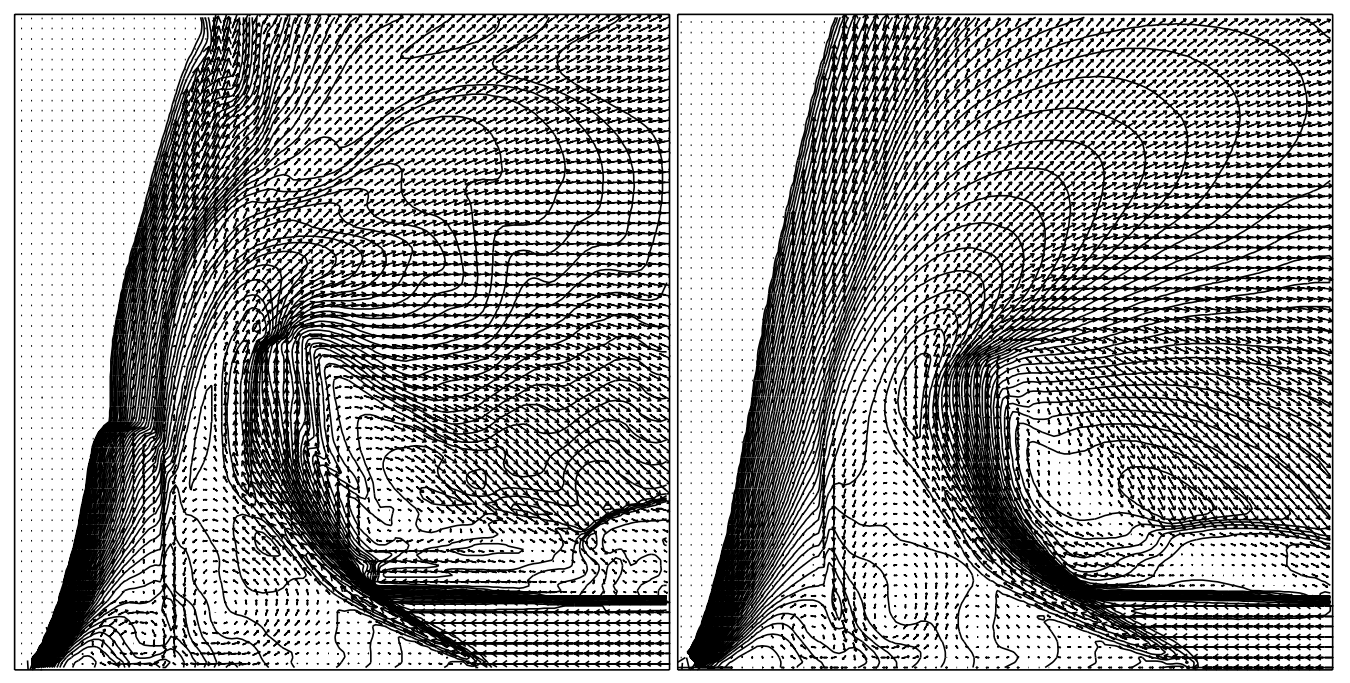

FIG. 5. - Same as Fig. 3, but for $t=2 \times 10^{4}$ in model M7 (left) and model M9 (right). The plots show the region with $0 \leq r \leq 50$ and $0 \leq z \leq 50$. 


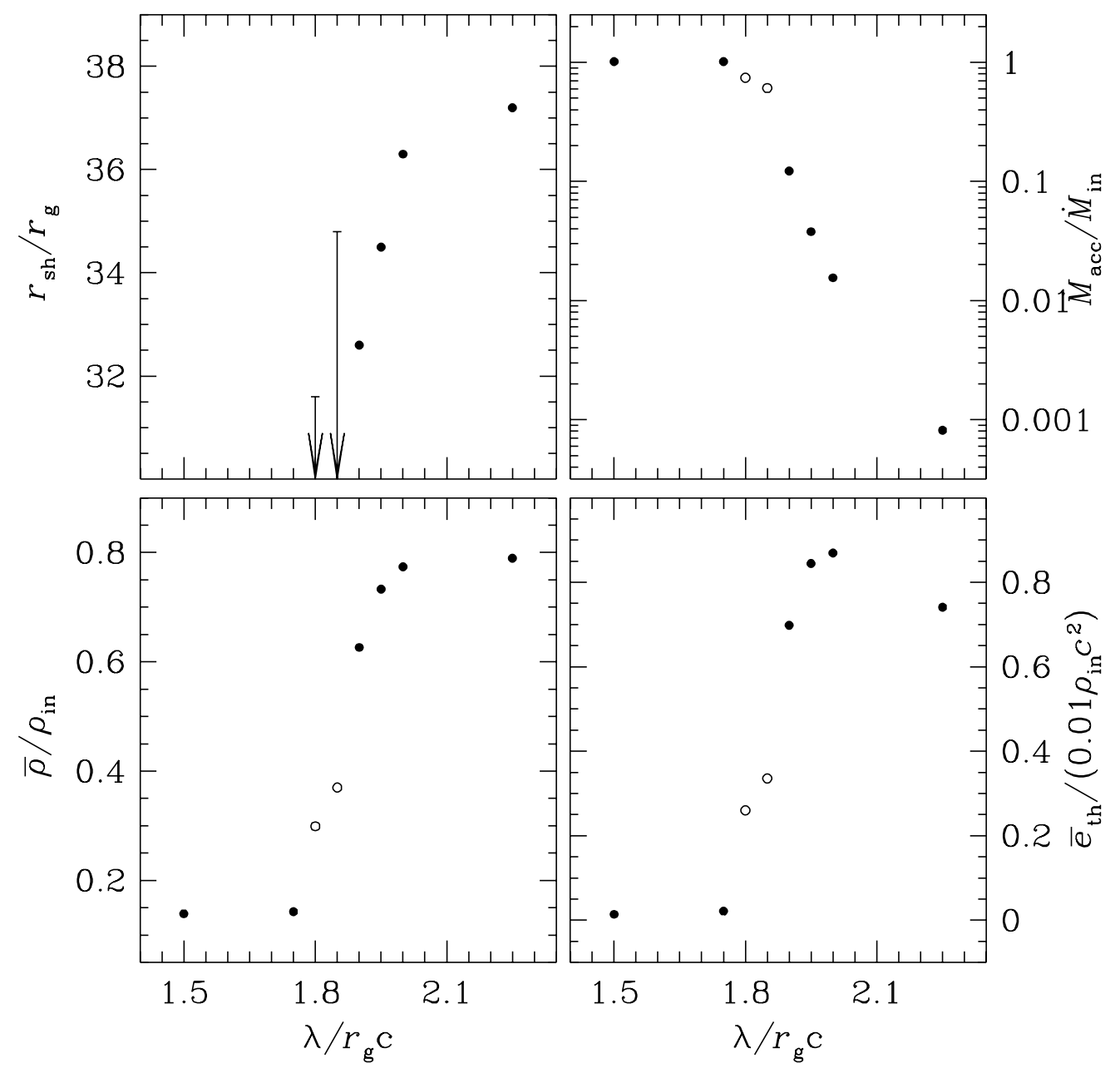

FIG. 6.-Time-averaged values of the position of the accretion shock along the equatorial plane, $r_{\text {sh }}$, the mass accretion rate into the black hole, $\dot{M}_{\text {acc }}$, the mean density, $\bar{\rho}$, and the mean thermal energy, $\bar{e}_{\mathrm{th}}$, of disk matter in models M1-M8. In the cases that show a periodic behavior (models M3 and M4), for the position of the accretion shock the largest value is plotted with arrows, and for other quantities the averaged values over exact periods are plotted with open circles.

(dashed curves) at four different phases in model M4 (at the same four epochs at which the panels of Fig. 4 were drawn). It is clear that the flow constantly tries to adjust itself to follow the analytic solution during the whole cycle. What actually happens is that the shock is never pressurebalanced, and the excess or deficit of total pressure continuously drives the shock to one direction or the other. Also, in the upper panel, the steady state solutions for $\lambda=1.9$ and 1.95 (long-dashed and long-dash-short-dashed curves, respectively) are shown. In these cases, the supersonic branch and the subsonic branch can be joined together to form a standing shock (indicated by vertical dotted lines at $r=13.1$ and 38.9, respectively), as described in Chakrabarti (1989) and Chakrabarti \& Molteni (1993). In the simulations (models M5 and M6), we do see a standing accretion shock as well. But the shock is located farther out, possibly because of the presence of vortex pressure, as discussed in Molteni et al. (1994). The bottommost solid curve, which crosses the $M=1$ line almost vertically, is for $\lambda=2$ and clearly does not have the inner sonic point. As a result, although the supersonic inflow can have a strong standing shock, matter does not accrete onto the black hole and must be deflected as winds.

\section{ASTROPHYSICAL IMPLICATIONS AND CONCLUDING REMARKS}

Accretion flows are expected to take place with various inflow conditions. In this paper, we have discussed the effects of varying angular momentum in thin, cold flows with vanishing net specific energy. We have divided the angular momentum space into four zones according to whether the flows allow sonic points and shocks. For the flows in zones I, III, and IV, stable solutions have been found. However, for the flows in zone II, numerical simulations have yielded an unstable solution. Here the flows periodically build and destroy the structure with the accretion shock on a timescale of several infall times. Two important properties of this zone point to its generic nature: (1) The range of angular momentum (from $\lambda_{b}=1.782$ to $\lambda_{u}=$ 1.854 for one-dimensional flows) is around the marginally stable value, $\lambda_{\mathrm{ms}}=1.837$, which is believed to be very natural for accretion flows. (2) In the limit of weak viscosity and negligible cooling (as we are considering here), our result is independent of the mass inflow rate.

The timescale of the periodicity of unstable flows is even more interesting. As noted in the previous section, it is in 


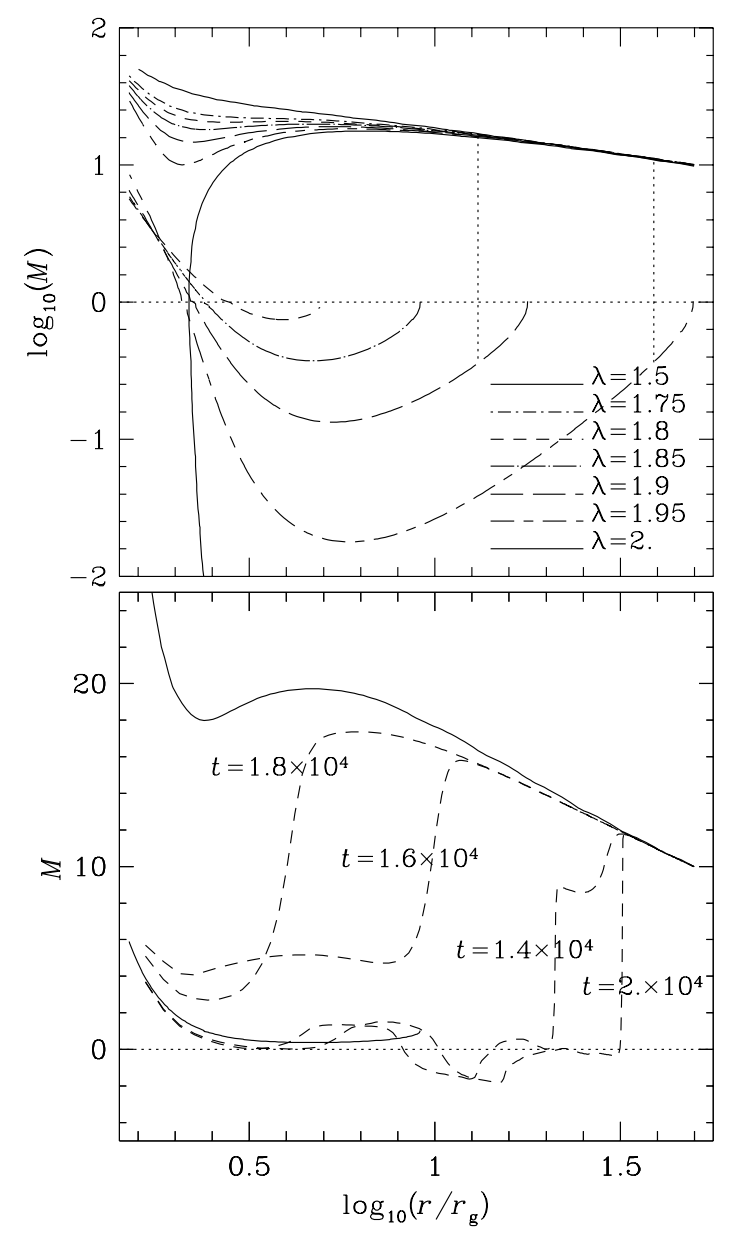

FIG. 7.-Top: Analytic solutions, Mach number vs. radial distance, of one-dimensional zero-energy flows with $M=10$ at $r=50$ whose angular momenta range from $\lambda=1.5$ to $\lambda=2$. Also, the positions of the stable accretion shocks are indicated with vertical dotted lines in the cases in which they exist (for $\lambda=1.9$ and 1.95). Bottom: Analytic Mach number distributions for $\lambda=1.85$ (solid curves), shown along with the numerical distributions on the equatorial plane (dashed curves) at four different phases in model M4.

the range of

$$
\tau \approx(4-6) \times 10^{3} \frac{R_{g}}{c}=(4-6) \times 10^{-2}\left(\frac{M_{\mathrm{bh}}}{1 M_{\odot}}\right) \mathrm{s} .
$$

The modulation of amplitude is also very significant and could be as much $100 \%$, depending on detailed processes. Oscillations with these characteristics have been observed in black hole candidates and are called QPOs. In the QPOs from the low-mass $\mathrm{X}$-ray binaries, the oscillation frequency has been found to lie typically between 5 and $60 \mathrm{~Hz}$ (van der Klis 1989; Dotani 1992; Mauche 1995, 1996). Thus compact objects with mass $M \approx 0.3-5 M_{\odot}$ could generate oscillations of right frequencies as a result of the instability discussed in this paper. Similarly, QPOs with a time period of $\sim 1$ day were reported in the Seyfert galaxy $\mathrm{RX}$ J0437.5-4711 (Halpern \& Marshall 1996), which also has the right frequency and energy range (extreme-UV) as we expect from the emission property of the postshock region. If, on the other hand, the oscillations are due to the shocks of the outflowing winds, the time period of oscillations would be shorter (by, say, an order of magnitude) since the corresponding stable shock locations in winds are closer to the black hole. However, considering the simplified physics that we have assumed here, it may be premature to assume that the presented mechanism could explain all the QPOs observed. As a future work, we plan to include radiative processes and viscosity in the accretion calculations, to examine the observational consequences of the present instability in more detail.

Note that there exist other mechanisms suggested to explain the QPOs. In particular, those for the multidimensional accretion flows include the dynamically induced instability suggested in Paper I and the thermally induced instability suggested in Molteni et al. (1996b). The first mechanism works if the accretion flow with $\lambda \gg \lambda_{\text {mb }}$ has a small inflow thickness, while the second works if the flow with nonnegligible cooling has a cooling timescale comparable to the dynamical timescale. As a result, the second depends on the accretion rate, while the first and the one in this paper are almost independent of the accretion rate (although indirect dependence comes in, as the region of parameter space that does not produce shocks itself depends on cooling processes). Particularly interesting is that the aforementioned oscillations of Molteni et al. (1996b) occur in the parameter space where a steady state analytic shock condition is satisfied, unlike the situations of models M3 and M4, in which the steady state shock condition is not satisfied. Recently, it has also been speculated that the acoustic perturbation of accretion shock waves (Yang \& Kafatos 1995) may cause QPO phenomena, although the amplitude of modulation may be smaller. It is possible that different observed QPOs may be due to one or more of these mechanisms.

The discussion presented here is valid for inviscid flows or flows with small viscosity. We believe that a small viscosity should not change our conclusions. As we have already discussed, with a larger viscosity, the steady state shock solutions disappear. It is not clear whether the same conclusions hold for the shocks in nonsteady solutions. Since the driving force that causes the oscillations is the imbalance between the total pressure on either side of the shock, it is quite likely that the oscillations will persist even in the presence of large viscosity. In the future, we plan to report the results of such a study.

D. R. thanks J. P. Ostriker for providing initial guidance of the work. The work by D. R. was supported in part by the Basic Science Research Institute Program, Korean Ministry of Education 1995, project BSRI-95-5408.

\section{REFERENCES}

Bondi, H. 1952, MNRAS, 112, 195

Chakrabarti, S. K. 1989, ApJ, 347, 365

1990, Theory of Transonic Astrophysical Flows (Singapore: World Sci.)

.1996, ApJ, 464, 664

Chakrabarti, S. K., \& Molteni, D. 1993, ApJ, 417, 671

. 1995, MNRAS, 272, 80
Chakrabarti, S. K., Ryu, D., \& Molteni, D. 1996, in preparation

Dotani, Y. 1992, in Frontiers of X-Ray Astronomy, ed. Y. Tanaka \&

K. Koyama (Tokyo: Universal Acad.), 152

Eggum, G. E., Coroniti, F. V., \& Katz, J. I. 1985, ApJ, 298, L41

Eggum, 1987, ApJ, 323, 634 1988, ApJ, 330, 142

Halpern, J. P., \& Marshall, H. 1996, ApJ, 464, 760 
Harten, A. 1983, J. Comput. Phys., 49, 357

Hawley, J. F. 1986, in IAUU Colloq. 89, Radiation Hydrodynamics in Stars and Compact Objects, ed. D. Mihalas \& K.-H. A. Winkler (Lecture Notes in Physics, 255) (Berlin: Springer), 369

Hawley, J. F., \& Smarr, L. L. 1986, in AIP Conf. Proc. 144, Magnetospheric Phenomena in Astrophysics, ed. R. Epstein (New York: AIP), 263

Hawley, J. F., Smarr, L. L., \& Wilson, J. R. 1984a, ApJ, 277, 296

Hawley, J. F., Smarr, L. L., \& Wilson, J. R. 1984b, ApJS, 55, 211

Igumenshchev, I. V., Chen, X., \& Abramowicz, M. A. 1996, MNRAS, 278, 236

Liang, E. P. T., \& Thompson, K. A. 1980, ApJ, 240, 271

Manmoto, T., Takeuchi, M., Mineshige, S., Matsumoto, R., \& Negoro, H. 1996, ApJ, 464, 135

Mauche, C. W. 1995, in IAU Colloq. 152, Astrophysics in the Extreme Ultraviolet, ed. S. C. Bowyer \& R. F. Malina (Dordrecht: Kluwer), 337
Mauche, C. W. 1996, ApJ, 463, L87

Molteni, D., Lanzafame, G., \& Chakrabarti, S. K. 1994, ApJ, 425, 161

Molteni, D., Ryu, D., \& Chakrabarti, S. K. 1996a, ApJ, 470, 460

Molteni, D., Sponholz, H., \& Chakrabarti, S. K. 1996b, ApJ, 457, 805

Narayan, R., \& Yi, I. 1994, ApJ, 428, L13

Paczyński, B., \& Wiita, P. J. 1980, A\&A, 88, 23

Rees, M. J., Begelman, M. C., Blandford, R. D., \& Phinney, E. S. 1982, Nature, 295, 17

Ryu, D., Brown, G. L., Ostriker, J. P., \& Loeb, A. 1995, ApJ, 452, 364 (Paper I)

Shapiro, S. L., \& Teukolsky, S. A. 1983, Black Holes, White Dwarfs, and Neutron Stars (New York: Wiley)

van der Klis, M. 1989, ARA\&A, 27, 517

Yang, Y., \& Kafatos, M. 1995, A\&A, 296, 238 